\title{
Situación, deficiencias y oportunidades de la administración y control de los recursos forestales y de fauna silvestre en el Perú, en el marco del proceso de descentralización y transferencia de funciones forestales
}

\author{
Situation, deficiencies and opportunities of the administration and control of \\ forest resources and wild fauna in Perú, within the framework of the process of \\ decentralization and transfer of forest functions
}

Giuliana N. Zamora-Velazco ${ }^{1, \star}$, Héctor E. Gonzales-Mora ${ }^{2}$ y María I. Manta-Nolasco ${ }^{3}$

\begin{tabular}{l} 
Recibido: 19 octubre 2020 | Aceptado: 05 mayo 2021 | Publicado en línea: 30 junio 2021 \\
Citación: Zamora-Velazco, GN; Gonzales-Mora, HE; Manta-Nolasco, MI. 2021. Situación, \\
deficiencias y oportunidades de la administración y control de los recursos forestales y de fauna \\
silvestre en el Perú, en el marco del proceso de descentralización y transferencia de funciones \\
forestales. Revista Forestal del Perú 36(1): 47-64. DOI: http://dx.doi.org/10.21704/rfp.vli36.1704 \\
\hline
\end{tabular}

\section{Resumen}

La presente investigación tiene por objetivo evaluar el desempeño de las entidades encargadas de la administración y control de los recursos forestales y de la fauna silvestre en el periodo 2012- 2018; a fin de determinar si el proceso de descentralización significó un avance en su gestión, así como, identificar debilidades y oportunidades de mejora en sus procesos. En función a ello se realizó: i) la caracterización del sector forestal a través de un análisis de Stakeholders; ii) la evaluación de la gestión de las entidades a través del presupuesto público ejecutado, presupuesto por resultados, proyectos de inversión y Sistema de Control Interno; y iii) análisis de deficiencias a partir de informes de control relacionados a la gestión de los Recursos Forestales y de Fauna Silvestre. Producto de lo señalado, se elaboró una matriz FODA y diagrama de verificación de riesgos para las Administraciones Técnicas Forestales y de Fauna Silvestre y Autoridades Regionales Forestales y de Fauna Silvestre. Se concluyó que las debilidades de las Administraciones Técnicas Forestales y de Fauna Silvestre radican en el poco interés del Servicio Nacional Forestal en fortalecer su gestión. A nivel normativo, el carácter temporal de las Administraciones Técnicas Forestales y de Fauna

\footnotetext{
${ }^{1}$ Órgano de Control Institucional, del Servicio Nacional de Áreas Naturales Protegidas por el Estado, Av. Gálvez Barrenechea N696, San Isidro, Lima, Perú.

${ }^{2}$ Departamento de Industrias Forestales, Facultad de Ciencias Forestales, Universidad Nacional Agraria La Molina, Av. La Molina s/n, La Molina, Lima, Perú.

${ }^{3}$ Departamento de Manejo Forestal, Facultad de Ciencias Forestales, Universidad Nacional Agraria La Molina, Av. La Molina s/n, La Molina, Lima, Perú.

* Autor de Correspondencia: giuliana.zamorav@gmail.com
} 
Silvestre reconocido en la Ley Forestal y de Fauna Silvestre N²9763. Las debilidades de las Autoridades Regionales Forestales y de Fauna Silvestre se encuentran ligadas al escaso presupuesto que le destinaron los Gobiernos Regionales; así como, a la falta de capacidad técnica. En conclusión, la descentralización de funciones forestales a los Gobiernos Regionales, en el periodo 2012-2018, no representó un avance en la gestión de los Recursos Forestales y de Fauna Silvestre; considerando el presupuesto, programas presupuestales e inversión pública; así como, las deficiencias de ejecución y funcionamiento; $y$, controles y revisiones en sus procesos.

Palabras clave: recursos forestales y de fauna silvestre, administración y control, descentralización, deficiencias, oportunidades de mejora

\begin{abstract}
The aim of this research is to assess the performance of the entities responsible for the administration and control of forest and wildlife resources for the period 2012-2018, in order to determine whether the decentralization process represents progress in their management, as well as identify weaknesses and possibilities for improvement in their processes. It was focused on: (i) the characterization of the forestry sector through an analysis of stakeholders; (ii) the evaluation of the entities financial year for which the public budget implemented was evaluated, budget by results, public investment projects and implementation of the Internal Control System; and (iii) Analysis of deficiencies about control reports related to the management of the forest and wildlife resources. On this basis, an SWOT matrix and risk verification diagram were developed for Forest and Wildlife Technical Administration and Regional Forestry Authority. It was concluded that the weaknesses of the Forest and Wildlife Technical Administration lie in the lack of interest of National Forest Service in strengthening their management. At the regulatory level, the lack of recognition of Forest and Wildlife Technical Administration in Forest and Wildlife Law N 29763. The weaknesses of the Regional Forestry Authority are linked to the scarce economic resources allocated by the Regional Governments, as well as the lack of technical capacity in the Regional Forestry Authority. In conclusion, the decentralization of forestry functions to the regional governments in the period 2012-2018 did not represent a step forward in the management of the forest and wildlife resources; considering the budget, budget programmes and public investment, as well as the deficiencies in implementation and operation; and, controls and revisions in its processes..
\end{abstract}

Key words: forest and wildlife resources, administration and control, decentralization, deficiencies, opportunities for improvement

\section{Introducción}

La descentralización es la distribución de competencias y funciones para el equilibrado uso del poder (Ley de Bases de la Descentralización n..$^{\circ} 27783$ ). La parte más importante del proceso de descentralización es la transferencia de competencias sectoriales, que consiste en el traslado de funciones y responsabilidades de los sectores del Gobierno Nacional, hacia los Gobiernos Sub Nacionales (CGR 2014).

Desde el año 2002 las funciones en materia agraria correspondientes a la gestión de los Re- cursos Forestales y de Fauna Silvestre (RFFS), comenzaron a ser transferidas de las Administraciones Técnicas Forestales y de Fauna Silvestre (ATFFS), órganos desconcentrados de la Dirección General Forestal y de Fauna Silvestre (DGFFS), del Ministerio de Agricultura, hacia los Gobiernos Regionales; para que estos se constituyan como Autoridad Regional Forestal y de Fauna Silvestre (ARFFS).

Sin embargo, este proceso no se concluyó, y a la fecha existen 15 departamentos, en los cuales aún existen ATFFS, organismos desconcentrados del Servicio Nacional Forestal- SERFOR 
(ex DGFFS) (MINAGRI 2019). Por otro lado, existen nueve Gobiernos Regionales que se han constituido como ARFFS.

Investigaciones similares en Chile y México revelaron que los procesos de descentralización se caracterizaron por la carencia de capital humano avanzado, la duplicidad de competencias y funciones, y la escasez de recursos financieros, debido a la fuerte centralización de los ingresos y de la decisión del gasto (Arredondo 2011). Así como, se resaltó la importancia de fortalecer la institucionalidad del Gobierno Central, e identificar los incentivos y peligros de la descentralización (Vidal 2004).

En este contexto, bajo la interrogante si la descentralización de funciones forestales fortaleció la institucionalidad forestal; y, si la conformación de las ARFFS mejoró la gestión de los RFFS del país; se planteó como objetivo general determinar si el proceso de descentralización, en el periodo 2012-2018, significó un avance en la gestión de los recursos forestales, así como establecer debilidades y oportunidades de mejora en sus procesos. Y siendo los objetivos específicos, i) caracterizar el sector forestal; ii) evaluar la gestión de las entidades encargadas de la administración y control de los RFFS a nivel de muestra, dos ARFFS y tres ATFFS en el ámbito de 24 departamentos, en el periodo 2012-2018; iii) identificar riesgos que ponen en peligro los fines y objetivos de la administración y control de los RFFS, delimitando responsabilidades de las entidades del sector; $y$, iv) determinar acciones a implementar para la mejora de la gestión de las ATFFS del SERFOR y las ARFFS.

\section{Materiales y Métodos}

\section{- Selección de Muestra}

Se seleccionó una muestra entre las ARFFS (9 departamentos) y las ATFFS (15 departamentos), en función a los criterios generales y específicos, siguientes:

Criterios generales: i) Condiciones de pobreza en las regiones (INEI 2016); ii) incidencia de conflictos socio ambientales (DP 2018); iii) índice de densidad del estado (PNUD 2013); y, iv) avance en el proceso de ordenamiento territorial (MINAM 2017).

Criterios específicos: i) Tasa de deforestación (MINAM 2015a); ii) extensión de bosques naturales (MINAM 2015b); iii) extensión de Bosques de producción permanente (MINAM 2015b); y, iv) incidencia de Ilegalidad en el Aprovechamiento Forestal (OSINFOR 2016).

Para cada criterio se seleccionaron los departamentos que tenían los tres mayores valores, en el caso de las ATFFS; mientras que, para las ARFFS, se seleccionó los dos departamentos con mayores valores. La muestra constó de las ATFFS de Cajamarca, Cusco y Pasco; así como, de las ARFFS de los Gobiernos Regionales de Loreto y Ucayali.

\section{- Metodología para el análisis de información}

A.1 Caracterización del sector forestal y de fauna silvestre

Se contó con información general como: normas de creación, Reglamento de Organización y Funciones (ROF) y otras normas que determinarán sus funciones específicas; para su uso en el Análisis de Stakeholders (TCU 2002), que mide cualitativamente los intereses de los grupos de interés con la finalidad de identificar y clasificar a los principales actores del sector forestal; $y$, cómo estos, influyen positiva o negativamente en el proceso de descentralización.

A.2 Evaluación del ejercicio de las entidades encargadas de la administración y control de los RFFS

Presupuesto ejecutado, periodo 2012- 2018 (MEF 2019a)

Antes de la creación del SERFOR, el presupuesto de la extinta DGFFS no diferenciaba su presupuesto a nivel de departamento, ni por ATFFS; por tanto, no se pudo realizar la búsqueda en los aplicativos del MEF. Recién a partir del 2015 existe información presupuestal del SERFOR y su ejecución por departamento; en tal sentido, la información se obtuvo del portal de Consulta Amigable del MEF (https://apps5.mineco.gob.pe/transparencia/ Navegador/). 
Para los Gobiernos Regionales de Loreto y Ucayali se tuvo en cuenta el Sistema Integrado de Administración Financiera del Estado (SIAF), y en él se buscó presupuesto ejecutado a nivel de la cadena presupuestal: pliego- programa y subprograma, a fin de identificar los subprogramas referidos a la gestión de los RFFS en cada Gobierno Regional.

Presupuesto por Resultados, periodo 20122018 (MEF 2019b)

Desde el año 2012, el sector forestal contó con un Programa Presupuestal- PP 84: "Manejo Eficiente de Recursos Forestales y Fauna Silvestre", posteriormente fue rediseñado el año 2015 creándose el PP 130: "Competitividad y aprovechamiento sostenible de los recursos forestales y de la fauna silvestre", que tiene como entidad responsable al SERFOR, e involucra a los tres niveles de Gobierno. Para su evaluación, se hizo referencia a ambos PP con la denominación del PP forestales, los datos se obtuvieron del portal de Consulta Amigable del MEF, en setiembre de 2019.

Estado de los Proyectos de Inversión- PI, periodo 2012-2018 (MEF 2019a)

Para las ATFFS en el periodo 2012-2014, se evaluaron los PI de la Unidad Ejecutora 001155: MINAGRI-Administración Central, cuando la entidad competente era la DGFFS del MINAGRI. A partir del año 2015, la búsqueda en la Consulta Amigable del MEF se efectuó en los PI del SERFOR. Y para las ARFFS, los PI correspondientes a los Gobiernos Regionales de Loreto y Ucayali.

\section{A.3 Implementación del SCI (CGR 2018)}

Se consideró la medición del grado de madurez del SCI efectuada por la Contraloría General de la República. (CGR) en el año 2016. Esta medición, determinó por niveles el Índice de implementación del SCI (Cuadro 1).

A.4 Análisis de deficiencias a partir de informes de control relacionados a la gestión de los RFFS, periodo 2012-2018 (CGR 2019)

Se analizó a partir de los informes de la CGR, el OCI del MINAGRI, y el OCI del SERFOR, en función a las deficiencias de las ATFFS y ARFFS seleccionadas, se tomaron las deficiencias, y se clasificaron, de acuerdo al detalle siguiente:

Deficiencias normativas: Casos en los que se identificó normas desactualizadas, ausencia de normas técnicas; o, regulación inadecuada de los procedimientos.

Deficiencias de organización: Casos en los que se identificó que el personal no cumplió con el perfil requerido; así como, en los que las entidades tenían una estructura orgánica no acorde con las funciones asignadas.

Deficiencias de ejecución y funcionamiento: Casos en los que se identificó inacción frente a las competencias, deficiencias en la

\begin{tabular}{|c|c|l|}
\hline Nivel & Índice & \multicolumn{1}{c|}{ Descripción } \\
\hline Inexistente & $0 \%$ & Las condiciones del elemento de control no existen. \\
\hline Inicial & $1 \%-20 \%$ & $\begin{array}{l}\text { Las condiciones del elemento de control existen, pero no están formaliza- } \\
\text { das. }\end{array}$ \\
\hline Intermedio & $21 \%-40 \%$ & Las condiciones del elemento de control existen y están formalizadas. \\
\hline Avanzado & $41 \%-60 \%$ & $\begin{array}{l}\text { Las condiciones del elemento de control están formalizadas, operando y } \\
\text { existe evidencia documental de su cumplimiento. }\end{array}$ \\
\hline Óptimo & $61 \%-80 \%$ & $\begin{array}{l}\text { Las condiciones del elemento de control están formalizadas, operando, } \\
\text { existe evidencia documental de su cumplimiento. }\end{array}$ \\
\hline Mejor continua & $81 \%-100 \%$ & $\begin{array}{l}\text { Las condiciones del elemento de control están formalizadas, operando y } \\
\text { existe evidencia documental de su cumplimiento y están en un proceso de } \\
\text { mejora continua. }\end{array}$ \\
\hline
\end{tabular}

Cuadro 1. Niveles e índice de implementación del SCI en las entidades del Estado. Fuente: Adaptado de Medición de la Implementación del Sistema de Control Interno en el Estado - Año 2014 (CGR 2015a). 
gestión de la información (registro, bases de datos, etc.), el incumplimiento de procedimientos técnicos o sancionadores, la ejecución de acciones sin contar con autorización o función; o, debilidades operativas y técnicas.

Deficiencias de controles y revisiones: Casos en los que se identificó la falta de supervisión; o, la supervisión insuficiente o negligente, de procedimientos o actividades.

A.5 Análisis matriz FODA y Diagrama de Verificación de Riesgos

El FODA se obtuvo de la información analizada y de los resultados obtenidos; así como, de la revisión bibliográfica recopilada. Como resultado se muestra esquemáticamente las fortalezas, debilidades, oportunidades y amenazas de la gestión de las ATFFS y ARFFS, dando como resultado una lista de pros y contras que ayudan en la toma de decisiones. El Diagrama de verificación de riesgos, es una matriz que mide cualitativamente la probabilidad e impacto potencial que tendrían los riesgos identificados, a partir de las debilidades y amenazas de los FODAS de las ATFFS y ARFFS.

\section{Resultados}

\section{A.1 Caracterización del sector forestal y de fauna silvestre}

\section{Análisis de Stakeholders}

Se identificó tres entidades y una dependencia con participación directa en el proceso de descentralización de funciones forestales hacía los Gobiernos Regionales. El MINAGRI a favor del proceso; siendo que las funciones a transferirse son del sector agricultura; el SERFOR, principal interesado (muy a favor) a fin de cumplir lo estipulado en la LFFS respecto a la organización del sector forestal; así como, dejar de tener bajo su dependencia y destinar recursos económicos a las ATFFS; y, el MINAM con un rol neutral. Las ATFFS, están en contra del proceso, toda vez que la descentralización no comprendería la transferencia del personal que labora en las ATFFS (Cuadro 2).

En tal sentido, se tienen dos tipos de Gobiernos Regionales; uno, en el ámbito de las ARFFS, los que a fin de fortalecer la gestión forestal descentralizada se encuentran a favor del proceso. El otro, en el ámbito de las ATFFS; los cuales estarían en contra del proceso, pues no recibirían una transferencia de recursos económicos ni técnicos; y pese a ello, tendría que asumir los costos de la gestión de los RFFS (Cuadro 3).

\section{A.2 Evaluación del ejercicio de las entidades encargadas de la administración y control de los RFFS}

Presupuesto ejecutado, periodo 2012- 2018

El presupuesto ejecutado por SERFOR en Cajamarca y Cusco, para el periodo evaluado, no superó el S/ 1500 000. En el 2018, la ejecución de Cajamarca y Cusco representó apenas el 1,2 \% del presupuesto que SERFOR ejecutó ese año (Figura 1). Cajamarca y Cusco

\begin{tabular}{|c|c|c|c|}
\hline Stakeholder & Posición & $\begin{array}{c}\text { Impacto si no cumple su } \\
\text { interés }\end{array}$ & Papel / Rol en el proceso \\
\hline MINAGRI & + A favor & Perder credibilidad & Ente rector de los RFFS \\
\hline SERFOR & ++ Muy a favor & Debilitaría la institucionalidad. & $\begin{array}{c}\text { Entidad técnico- normativa del } \\
\text { sector forestal }\end{array}$ \\
\hline ATFFS & - En contra & $\begin{array}{c}\text { Continuar ejerciendo la } \\
\text { gestión de los RFFS. }\end{array}$ & $\begin{array}{c}\text { Organismos Desconcentrados del } \\
\text { SERFOR }\end{array}$ \\
\hline MINAM & 0 Neutral & $\begin{array}{c}\text { Asumir un rol protagónico en } \\
\text { la gestión de bosques. }\end{array}$ & $\begin{array}{c}\text { Asegurar el uso racional y ético } \\
\text { de los RRNN y del medio am- } \\
\text { biente }\end{array}$ \\
\hline
\end{tabular}

Cuadro 2. Análisis de Stakeholders de las entidades y dependencias del sector forestal del Gobierno Nacional. 


\begin{tabular}{|c|c|c|c|}
\hline Stakeholder & Posición & $\begin{array}{c}\text { Impacto si no se cumple su } \\
\text { interés }\end{array}$ & Papel / Rol en el proceso \\
\hline ARFFS & + A favor & $\begin{array}{c}\text { Aumento de los índices de } \\
\text { ilegalidad en dichas regiones. }\end{array}$ & $\begin{array}{c}\text { Dependencia administradora } \\
\text { y de Control de los RFFS. }\end{array}$ \\
\hline $\begin{array}{c}\text { Gobiernos Regionales en el } \\
\text { ámbito de las ATFFS }\end{array}$ & - En contra & $\begin{array}{c}\text { Perdida de la autonomía en } \\
\text { relación con la gestión de los } \\
\text { RFFS de dichas regiones. }\end{array}$ & $\begin{array}{c}\text { Entidad con competencias en } \\
\text { materia forestal, que aún no } \\
\text { las viene ejerciendo. }\end{array}$ \\
\hline
\end{tabular}

Cuadro 3. Análisis de Stakeholders de los Gobiernos Regionales.

\begin{tabular}{|c|c|c|c|c|c|c|}
\hline \multirow[b]{2}{*}{ Año } & \multicolumn{3}{|c|}{ Gobierno Regional de Loreto } & \multicolumn{3}{|c|}{ Gobierno Regional de Ucayali } \\
\hline & 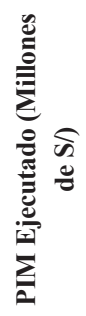 &  & 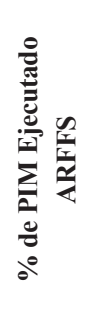 & 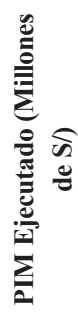 &  & 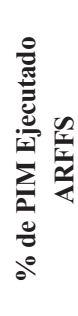 \\
\hline 2012 & 1178 & 0,4 & $0,03 \%$ & 466 & 2 & $0,5 \%$ \\
\hline 2013 & 1211 & 0,8 & $0,1 \%$ & 565 & 3 & $0,5 \%$ \\
\hline 2014 & 1304 & 2 & $0,1 \%$ & 596 & 5 & $1 \%$ \\
\hline 2015 & 1232 & 3 & $0,2 \%$ & 606 & 7 & $1 \%$ \\
\hline 2016 & 1335 & 2 & $0,1 \%$ & 754 & 7 & $1 \%$ \\
\hline 2017 & 1618 & 0,5 & $0,02 \%$ & 849 & 4 & $0,5 \%$ \\
\hline 2018 & 1714 & 2 & $0,1 \%$ & 861 & 5 & $0,6 \%$ \\
\hline
\end{tabular}

Cuadro 4. Presupuesto ejecutado por los Gobiernos Regionales de Loreto y Ucayali y sus ARFFS. Fuente: Elaborado con base en la Consulta amigable: ejecución del gasto por pliego y departamento (MEF 2019a).

\begin{tabular}{|c|c|c|c|c|c|c|}
\hline \multirow{2}{*}{ Año } & \multicolumn{2}{|c|}{ Cajamarca } & \multicolumn{2}{c|}{ Cusco } & \multicolumn{2}{c|}{ Pasco } \\
\cline { 2 - 7 } & $\begin{array}{c}\text { Presupuesto } \\
\text { ejecutado en } \\
\text { el PP Forestal }\end{array}$ & $\begin{array}{c}\text { \% Total } \\
\text { ejecutado en } \\
\text { PP por } \\
\text { SERFOR }\end{array}$ & $\begin{array}{c}\text { Presupuesto } \\
\text { ejecutado en } \\
\text { el PP Forestal }\end{array}$ & $\begin{array}{c}\text { \% Total } \\
\text { ejecutado en } \\
\text { PP por } \\
\text { SERFOR }\end{array}$ & $\begin{array}{c}\text { Presupuesto } \\
\text { ejecutado en } \\
\text { el PP Forestal }\end{array}$ & $\begin{array}{c}\text { Tocecutad } \\
\text { PP por } \\
\text { SERFOR }\end{array}$ \\
\hline 2012 & 0 & 0 & 0 & 0 & 0 & 0 \\
\hline 2013 & 0 & 0 & 218646 & $1 \%$ & 0 & 0 \\
\hline 2014 & 673935 & $2 \%$ & 667147 & $2 \%$ & 0 & 0 \\
\hline 2015 & 879268 & $2 \%$ & 925881 & $2 \%$ & 0 & 0 \\
\hline 2016 & 1192779 & $2 \%$ & 1130857 & $2 \%$ & 396996 & $1 \%$ \\
\hline 2017 & 1284535 & $2 \%$ & 1221537 & $2 \%$ & 63924 & $0,1 \%$ \\
\hline 2018 & 1161539 & $1,4 \%$ & 1205635 & $1,5 \%$ & 100607 & $0,001 \%$ \\
\hline
\end{tabular}

Cuadro 5. Presupuesto ejecutado en los PP forestales de Cajamarca, Cusco y Pasco. Fuente: Elaborado con base en la Consulta amigable: ejecución del gasto por programa presupuestal y entidad (MEF 2019b). 


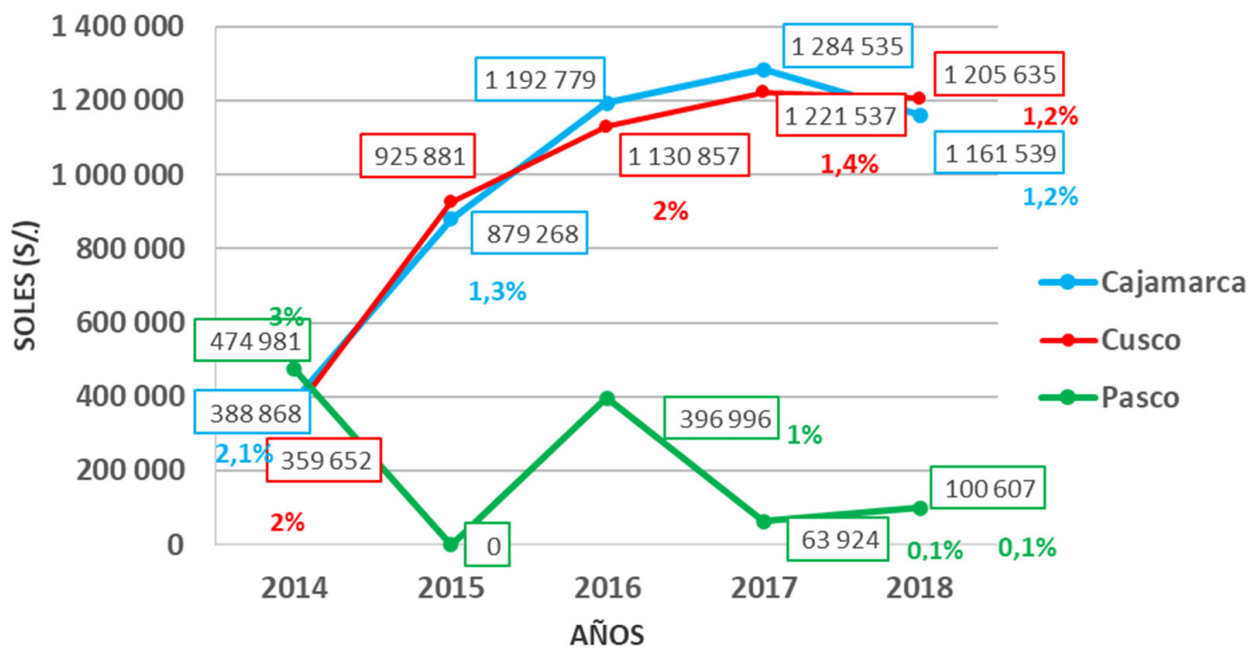

Figura 1. Presupuesto ejecutado por el SERFOR en Cajamarca, Cusco y Pasco.

cuentan con bajos presupuestos, pese a que la gestión de los RFFS en ambos departamentos se realiza a través de una ATFFS.

En Pasco, el presupuesto ejecutado fue disminuyendo del 2014 al 2017; a pesar del incremento presupuestal del 2017 al 2018, la representatividad respecto a lo ejecutado por SERFOR fue de $0,1 \%$.

Por otro lado, el presupuesto que ejecutó la ARFFS de Loreto en el periodo 2012-2018 (Cuadro 4), no superó el 0,5 \% del total ejecutado por el Gobierno Regional. Si bien el Gobierno Regional de Loreto incrementó su ejecución presupuestal en el periodo 2012-2018, eso no ocurrió con la ARFFS Loreto. Ucayali destinó mayor presupuesto que Loreto en la gestión de los RFFS (Cuadro 4), siendo que su representatividad con respecto del total ejecutado por el Gobierno Regional fluctuó entre 0,5 y $1 \%$, en el periodo 2012-2018. Si bien el Gobierno Regional de Ucayali incrementó su ejecución presupuestal, eso no ocurrió con su ARFFS.

Presupuesto por Resultados, periodo 20122018 (MEF 2019b)

En Cajamarca y Cusco el presupuesto bajo PP alcanzó como máximo el 2\% del presupues- to que ejecutó SERFOR en el PP forestal. Pasco, no hizo uso de PP forestal en el periodo 20122015; siendo que en el año 2018 alcanzó una representatividad de $0,001 \%$. (Cuadro 5).

Para las ARFFS de Loreto y Ucayali se comparó su ejecución en el PP forestal, respecto al presupuesto ejecutado por todos los Gobiernos Regionales en el marco del PP forestal, para los años 2012-2018. Para la ARFFS de Loreto, se obtuvo que sólo en los años 2015 y 2018 el presupuesto en el PP forestal superó el millón de soles. En contraste, la ARFFS Ucayali en los años 2016 y 2017 ejecutó más de 2 millones de soles en el PP forestal; y en el 2018 superó los 4 millones (Cuadro 6).

Estado de los Proyectos de Inversión- PI, periodo 2012-2018 (MEF 2019a)

SERFOR no ejecutó PI relacionados a la gestión de los RFFS en Cusco y Cajamarca. En Pasco, SERFOR vienen ejecutando cuatro PI que lo contemplan como beneficiario (entre otros departamentos); de estos cuatro PI, en el periodo evaluado, sólo dos ejecutaron recursos en Pasco, por un total de S/ 561527.

Entre el 2012 y 2018, el Gobierno Regional de Loreto ejecutó cuatro PI relacionados a 
Situación, deficiencias y oportunidades de la administración de los recursos forestales y de fauna silvestre en el Perú

\begin{tabular}{|c|c|c|c|c|}
\hline \multirow[b]{2}{*}{ Año } & \multicolumn{2}{|c|}{ Gobierno Regional Loreto } & \multicolumn{2}{|c|}{ Gobierno Regional Ucayali } \\
\hline & $\begin{array}{c}\text { Presupuesto } \\
\text { ejecutado PP } \\
\text { Forestal }\end{array}$ & $\begin{array}{c}\% \text { del total } \\
\text { ejecutado en PP }\end{array}$ & $\begin{array}{c}\text { Presupuesto } \\
\text { ejecutado PP } \\
\text { Forestal }\end{array}$ & $\begin{array}{c}\text { \% del total } \\
\text { ejecutado en PP }\end{array}$ \\
\hline 2012 & - & - & - & - \\
\hline 2013 & 15750 & $0,3 \%$ & - & - \\
\hline 2014 & 100000 & $1 \%$ & 38962 & $0,3 \%$ \\
\hline 2015 & 1374302 & $10 \%$ & 2000 & $0.01 \%$ \\
\hline 2016 & 538232 & $4 \%$ & 2998472 & $20 \%$ \\
\hline 2017 & 301850 & $2 \%$ & 2674005 & $19 \%$ \\
\hline 2018 & 1449536 & $6 \%$ & 4030662 & $16 \%$ \\
\hline
\end{tabular}

Cuadro 6. Presupuesto ejecutado por las ARFFS de Loreto y Ucayali en el PP forestal. Fuente: Elaborado con base en la Consulta amigable: ejecución del gasto por programa presupuestal y entidad (MEF 2019b).

\begin{tabular}{|c|c|c|}
\hline Entidad & Puntaje Total (\%) & Nivel Maduración \\
\hline SERFOR & 28,98 & Intermedio \\
\hline $\begin{array}{c}\text { Gobierno Regional de } \\
\text { Loreto }\end{array}$ & 11,94 & Inicial \\
\hline $\begin{array}{c}\text { Gobierno Regional de } \\
\text { Ucayali }\end{array}$ & 24,03 & Intermedio \\
\hline
\end{tabular}

Cuadro 7. Madurez del SCI- Año 2016. Fuente: Elaborado con base en la consulta efectuada por transparencia sobre el grado de madurez del Sistema de Control Interno de SERFOR y de los Gobiernos Regionales de Loreto y Ucayali (CGR 2018).

la gestión de los RFFS por un monto de S/ 3 480 669. Por su parte, el Gobierno Regional de Ucayali ejecutó ocho PI, por un monto de S/ 12 937 542. Cabe agregar, que los montos ejecutados en PI de RRFS por las ARFFS de Loreto y Ucayali, no supero el 1\% y $2 \%$; respectivamente, en comparación con el presupuesto ejecutado en PI por estos Gobiernos Regionales (MEF 2019a).

\section{A.3 Implementación del SCI (CGR 2018)}

El SERFOR y el Gobierno Regional de Ucayali se encuentran en un nivel de maduración intermedio; es decir, las condiciones del elemento de control existen y están formalizadas. El Gobierno Regional de Loreto se encuentra en un nivel inicial; es decir, las condiciones del elemento de control existen, pero no están formalizadas (Cuadro 7). Por tanto, el Gobierno Regional de Loreto es más sensible a la existencia de prácticas corruptas en su organización.

\section{A.4 Análisis de deficiencias de informes de con- trol sobre la gestión de los RFFS (CGR 2019)}

\section{Deficiencias de las ATFFS seleccionadas}

En el periodo 2012-2018 se emitieron nueve informes cuya materia a examinar fue la gestión de las ATFFS de la muestra seleccionada. Los informes evidenciaron 72 deficiencias, relacionadas en 51 casos a Ejecución y Funcionamiento, 15 a Controles y Revisiones; y finalmente, cinco Normativas (Cuadro 8).

\section{Deficiencias de las ARFFS seleccionadas}

Con respecto a la gestión de los Gobiernos Regionales de Ucayali y Loreto en su rol de ARFFS se emitieron 30 informes. Los informes evidenciaron 34 deficiencias relacionadas en 27 casos a deficiencias de ejecución y funcionamiento, cinco a controles y revisiones; y finalmente, y dos a deficiencias de organización (Cuadro 8). 


\begin{tabular}{|c|c|c|c|}
\hline Deficiencias & Descripción & $\begin{array}{c}\mathrm{N}^{\circ} \text { de } \\
\text { deficiencias } \\
\text { en ATFFS }\end{array}$ & $\begin{array}{l}\mathrm{N}^{\circ} \text { de } \\
\text { deficiencias } \\
\text { en ARFFS }\end{array}$ \\
\hline \multirow{3}{*}{$\begin{array}{c}\text { Deficiencias de Organización } \\
\text { (ATFFS: 5) } \\
\text { (ARFFS: } 2)\end{array}$} & $\begin{array}{l}\text { Ausencia de articulación entre las Unida- } \\
\text { des Orgánicas encargadas de una misma } \\
\text { función al interior de la entidad }\end{array}$ & 1 & 0 \\
\hline & $\begin{array}{l}\text { Personal que no cumple con el perfil } \\
\text { requerido o establecido en el Manual de } \\
\text { Organización y Funciones- MOF de la } \\
\text { entidad }\end{array}$ & 2 & 1 \\
\hline & $\begin{array}{l}\text { Entidades con una estructura orgánica no } \\
\text { acorde con las funciones asignadas }\end{array}$ & 2 & 1 \\
\hline \multirow{5}{*}{$\begin{array}{l}\text { Deficiencias de Ejecución y } \\
\text { Funcionamiento } \\
\text { (ATFFS: } 51 \text { ) } \\
\text { (ARFFS: 27) }\end{array}$} & Inacción frente a sus competencias & 7 & 2 \\
\hline & $\begin{array}{l}\text { Débil gestión de la información (inven- } \\
\text { tarios, diagnósticos, registros, bases de } \\
\text { datos, entre otros.) }\end{array}$ & 10 & 1 \\
\hline & $\begin{array}{l}\text { Incumplimiento de procedimiento admi- } \\
\text { nistrativo sancionador o técnico debida- } \\
\text { mente aprobado }\end{array}$ & 26 & 23 \\
\hline & $\begin{array}{l}\text { Ejecución de acciones sin contar con la } \\
\text { debida autorización, no sustentarse en una } \\
\text { función o competencia. }\end{array}$ & 3 & 0 \\
\hline & $\begin{array}{l}\text { Debilidades operativas y técnicas limitan } \\
\text { el logro de objetivos y metas }\end{array}$ & 5 & 1 \\
\hline \multirow{2}{*}{$\begin{array}{l}\text { Deficiencias de Controles y } \\
\text { Revisiones } \\
\text { (ATFFS: 15) } \\
\text { (ARFFS: 5) }\end{array}$} & $\begin{array}{l}\text { Ausencia de supervisión o monitoreo de } \\
\text { procedimientos o actividades }\end{array}$ & 5 & 0 \\
\hline & $\begin{array}{l}\text { Insuficiente o negligente supervisión o } \\
\text { monitoreo de procedimientos o actividades }\end{array}$ & 10 & 5 \\
\hline
\end{tabular}

Cuadro 8. Análisis de deficiencias de la gestión de los RFFS en las ATFFS y ARFFS.

\section{A.5 Análisis FODA}

\section{ATFFS}

Como fortalezas se tiene que las ATFFS cuentan con el respaldo técnico del SERFOR, al ser sus órganos desconcentrados y tener una dependencia funcional; esto también facilita las coordinaciones interinstitucionales con otras entidades del Gobierno Nacional como son el SERNANP, OSINFOR, OEFA, entre otras.

Las oportunidades están ligadas a su dependencia del SERFOR, a través de la cual pueden tener mayores facilidades para captar recursos de la cooperación internacional; y el encontrarse en zonas donde no hay abundancia de RFFS existe una menor presión de actividades ilegales en el ámbito de su jurisdicción.
Las debilidades identificadas radican en el poco interés del SERFOR en fortalecer la gestión de las ATFFS; en tal sentido, destinan escasos recursos económicos para su gestión. Esto último se refleja en la incipiente representatividad que tienen en el presupuesto por resultados que ejecuta el SERFOR, y estar apenas contemplados en los PI que desarrolla dicha entidad.

A nivel normativo, la falta de reconocimiento en la LFFS N²9763 de las ATFFS, bajo el entendido que el proceso de descentralización ya se culminó del todo.

Asimismo, se identificó como debilidad la insuficiente o negligente supervisión o monitoreo de procedimientos o actividades, la limitada implementación del SCI en el SERFOR; 


\begin{tabular}{|c|c|}
\hline Fortalezas & Oportunidades \\
\hline $\begin{array}{l}\text { - } \quad \text { Respaldo técnico de la Autoridad Forestal } \\
\text { Nacional- SERFOR. } \\
\text { - } \quad \text { Facilidades para la gestión interinstitucional. }\end{array}$ & $\begin{array}{l}\text { - Cooperación internacional captada por su } \\
\text { dependencia del SERFOR. } \\
\text { Menor presión sobre los RFFS al ser estos } \\
\text { menos abundantes. }\end{array}$ \\
\hline Debilidades & Amenazas \\
\hline $\begin{array}{l}\text { Poco interés del SERFOR en fortalecer su } \\
\text { gestión. } \\
\text { - } \quad \text { Presupuesto escaso en las ATFFS. } \\
\text { - } \quad \text { La LFFS vigente no reconoce a las ATFFS. } \\
\text { - } \quad \text { Escasa ejecución del PP en las ATFFS. } \\
\text { Limitada y deficiente gestión de PI en } \\
\text { ATFFS. } \\
\text { - } \quad \text { Limitada implementación del SCI en el } \\
\text { - } \quad \text { Insuficiente o negligente supervisión o moni- } \\
\text { toreo de procedimientos o actividades. } \\
\text { Débil gestión de la información (inventarios, } \\
\text { diagnósticos, registros, bases de datos). } \\
\text { Incumplimiento de procedimiento adminis- } \\
\text { trativo sancionador o técnico. }\end{array}$ & $\begin{array}{l}\text { Débil proceso de descentralización a nivel } \\
\text { nacional. } \\
\text { Presión por minería y cambio de uso de la } \\
\text { tierra a agricultura en menor escala. } \\
\text { - Limitado avance del proceso de ordenamien- } \\
\text { to territorial a nivel nacional. }\end{array}$ \\
\hline
\end{tabular}

Cuadro 9. Matriz FODA de las ATFFS del SERFOR. Fuente: Elaborado con base en los resultados obtenidos del análisis y revisión bibliográfica.

\begin{tabular}{|c|c|}
\hline Fortalezas & Oportunidades \\
\hline $\begin{array}{l}\text { - Autonomía administrativa de los GR para } \\
\text { organizarse internamente y reglamentar los } \\
\text { servicios públicos a su cargo. } \\
\text { - Funciones claramente asignadas en cuanto a } \\
\text { la gestión de los RFFS. }\end{array}$ & $\begin{array}{l}\text { - Cooperación internacional principalmente } \\
\text { interesada en apoyar la gestión forestal } \\
\text { sostenible. } \\
\text { - Creciente demanda de productos forestales } \\
\text { bajo certificación forestal. } \\
\text { - Convenios y acuerdos internacionales que } \\
\text { fomentan el manejo sostenible y trazable de } \\
\text { los RFFS. }\end{array}$ \\
\hline Debilidades & Amenazas \\
\hline $\begin{array}{l}\text { - } \\
\text { - } \quad \text { Limitada y deficiente gestión de PI en ARFFS. } \\
\text { - Limitada implementación del SCI en los GR. } \\
\text { - } \quad \text { Insuficiente o negligente supervisión o moni- } \\
\text { toreo de procedimientos o actividades. } \\
\text { - Incumplimiento de procedimiento adminis- } \\
\text { trativo sancionador o técnico. } \\
\text { - Poca capacidad de coordinación interinsti- } \\
\text { tucional. } \\
\text { - Falta de capacidad técnica. }\end{array}$ & $\begin{array}{l}\text { El creciente mercado de los biocombustibles, } \\
\text { principalmente por la palma aceitera. } \\
\text { - El desarrollo de megaproyectos (construcción } \\
\text { de hidroeléctricas y/o carreteras). } \\
\text { - Limitado avance del proceso de ordenamien- } \\
\text { to territorial a nivel nacional. } \\
\text { - Evidencias de prácticas corruptas en el mane- } \\
\text { jo forestal. } \\
\text { - Débil proceso de descentralización a nivel } \\
\text { nacional. } \\
\text { - Falta de empoderamiento de los GR. }\end{array}$ \\
\hline
\end{tabular}

Cuadro 10 . Matriz FODA de las ARFFS. Fuente: Elaborado con base en los resultados obtenidos del análisis y revisión bibliográfica. 
la débil gestión de la información (inventarios, diagnósticos, registros, bases de datos, entre otros; así como el incumplimiento por parte de sus servidores de procedimientos administrativos sancionadores o técnicos debidamente aprobado.

Como amenazas, se identificó el débil proceso de descentralización a nivel nacional que podría ocasionar que estos órganos se establezcan de forma perenne, la presión por minería y cambio de uso de la tierra a agricultura en menor escala y el limitado avance del proceso de ordenamiento territorial a nivel nacional (Cuadro 9).

\section{ARFFS}

Como fortalezas se identificó la autonomía administrativa de los Gobiernos Regionales para organizarse internamente y reglamentar los servicios públicos a su cargo; así como, el hecho de contar con resoluciones ministeriales que expresamente le designan funciones en materia forestal.

Como oportunidades se considera a la posibilidad de contar con recursos de cooperación internacional principalmente interesada en apoyar la gestión forestal de las ARFFS; y, la creciente demanda de productos forestales maderables y no maderables, bajo certificación forestal; así como, los convenios, acuerdos y cooperantes internacionales que fomentan el manejo sostenible y trazable de los RFFS.

De las debilidades identificadas, siete en total, se encuentran ligadas a los escasos recursos económicos que destinan los Gobiernos Regionales en la gestión de los RFFS que ejecutan las ARFFS; así como, la falta de capacidad técnica en las ARFFS lo cual se refleja en la deficiente gestión de PI relacionados a los RFFS.

Sumado a ello, se evidenció, la insuficiente o negligente supervisión o monitoreo de procedimientos o actividades en las ARFFS; el incumplimiento de procedimientos administrativos sancionadores o técnicos debidamente aprobados. Y en un sentido más general, se observó poca capacidad de coordinación interinstitucional; y la limitada implementación del SCI en los Gobiernos Regionales.
Las amenazas son el creciente mercado de los biocombustibles que genera presión sobre los bosques y el avance del cambio de uso de la tierra, principalmente por la palma aceitera; el desarrollo de megaproyectos como la construcción de hidroeléctricas que vienen amenazando la amazonia. Así también otros factores más generales como: el limitado avance del proceso de ordenamiento territorial a nivel nacional; el débil proceso de descentralización a nivel nacional; y la falta de empoderamiento de los Gobiernos Regionales. Finalmente, otra amenaza la configuran las evidencias de prácticas corruptas en el manejo forestal en el ámbito de las ARFFS.

\section{A.6 Identificación de Riesgos}

A partir de las Debilidades y Amenazas identificadas en las matrices FODA de las ATFFS y ARFFS se identificaron nueve riesgos; que se listan a continuación: R1: Limitado desarrollo de la producción forestal; R2: Deficientes capacidades técnicas; o logísticas de la gestión forestal; R3: Pérdida de la cobertura forestal; R4: Desconocimiento de los objetivos de la gestión forestal; R5: Débil institucionalidad; R6: Incremento de las prácticas corruptas en la gestión forestal; R7: Incremento del transporte ilegal de RFFS; R8: Desconocimiento de la realidad de la producción forestal; $y, R 9$ : Desconocimiento de la información y apoyo interinstitucional en el control forestal (Cuadro 10).

\section{A.7 Diagrama de Verificación de Riesgos}

La distribución de los nueve riesgos identificados de acuerdo con su probabilidad de ocurrencia e impacto varía para las ATFFS y ARFFS. En el caso de las ATFFS los riesgos en el cuadrante de alta probabilidad e impacto son seis, presenta dos riesgos en el cuadrante de alto impacto y baja probabilidad de ocurrencia y un riesgo en el cuadrante de bajo impacto y baja probabilidad de ocurrencia (Figura 2).

Para las ARFFS los nueve riesgos se ubican en el cuadrante de alta probabilidad e impacto; ello debido a que albergan mayor cantidad y riqueza los RFFS a nivel nacional (Figura 2). 


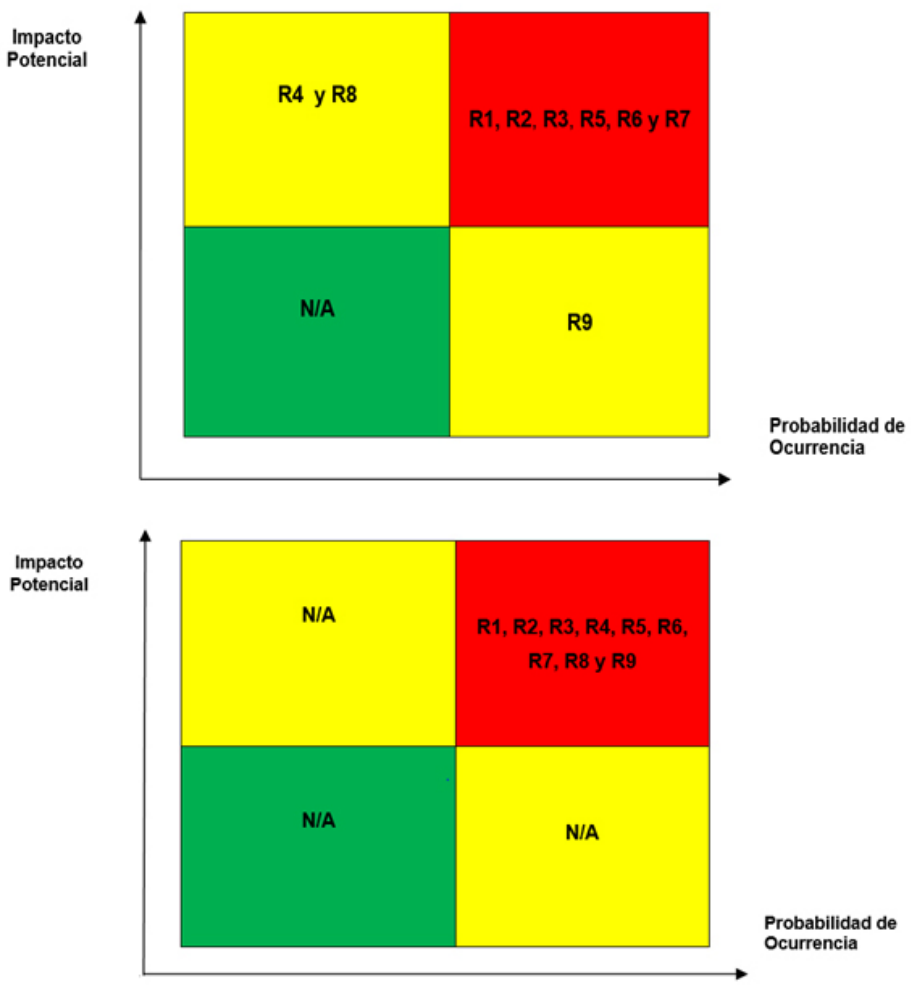

Figura 2 . Diagrama de verificación de riesgos de las ATFFS (superior) y ARFFS (inferior).

\section{Discusión}

\section{Caracterización del sector forestal y de fauna silvestre}

El SERFOR es la entidad principalmente interesada (muy a favor) en culminar el proceso de transferencia de funciones forestales; por tanto, debe planificar y programar dentro de su plan operativo institucional, la asistencia técnica a los Gobiernos Regionales que aún no han culminado la transferencia de funciones, a fin de fortalecer sus capacidades; y de esta manera minimizar su oposición en contra a la transferencia de funciones. Dado que, si bien es cierto, el proceso de descentralización del país no comprende la transferencia de recursos económicos, logísticos ni técnicos, se puede asistir técnicamente la modificación de norma- tiva interna tales como, el Texto Único de Procedimientos Administrativos (TUPA) y el ROF de estos Gobiernos Regionales.

Al respecto, en el 2017, el MINAGRI aprobó su Plan de Transferencia de competencias sectoriales a los Gobiernos Regionales y Locales del año 2017 (MINAGRI 2017). En dicho Plan de Transferencia SERFOR identificó que lo pendiente correspondía a la adecuación de los instrumentos de gestión (TUPA y ROF) a la LFFS y sus Reglamentos para los quince Gobiernos Regionales pendientes de recibir funciones (MINAGRI 2017).

En el año 2019 el Plan de Transferencia de competencias del MINAGRI a los Gobiernos Regionales y Gobiernos Locales, se incorporó nuevamente las funciones e) y q) del artículo 
$51^{\circ}$ de la Ley Orgánica de Gobiernos Regionales, para los 15 Gobiernos Regionales pendientes de recibir funciones (MINAGRI 2019), lo que pone en manifiesto la intención política del Sector Agricultura en culminar esta transferencia de funciones, pese a la desidia de los Gobiernos Regionales que deben recibirlas.

$\mathrm{Al}$ año 2019 MINAGRI informa que ha transferido el 92\% de las Funciones en Materia Agraria que incluye al artículo $51^{\circ}$ de la Ley Orgánica de Gobiernos Regionales (MINAGRI 2019). Situación que deja en evidencia la tarea pendiente respecto a la descentralización por parte de las autoridades competentes.

Cabe agregar, que en la administración de los recursos forestales de otros países de la región también se aplica una descentralización de funciones; por ejemplo, en Colombia, el ente rector de la gestión de los recursos forestales es el Ministerio de ambiente, vivienda y desarrollo territorial creado en el año 1993, y desde el 2011 denominado Ministerio del Ambiente y Desarrollo Sostenible (MINAMBIENTE 2021). El Ministerio de agricultura y desarrollo rural sólo tiene el rol rector de la producción forestal.

Asimismo, en el ámbito de 32 departamentos existen 27 Corporaciones Autónomas Regionales y de Desarrollo Sostenible que cuentan con Dependencias Especiales Forestales, cuyo accionar se basa en las directrices del Ministerio de ambiente, vivienda y desarrollo territorial. Es decir, el Ministerio del Ambiente concentra las funciones forestales, sólo dejándole al de Agricultura la de rector de la producción forestal; y a su vez, tiene sus funciones descentralizadas a través de las Corporaciones Autónomas Regionales y de Desarrollo Sostenible (DAFP 2018).

Por otro lado, en Brasil como un estado federal, las competencias forestales se encuentran concentradas en el Ministerio de Medio Ambiente, a través del Servicio Forestal Brasileño creado en el 2006, y cuya competencia exclusiva es en los bosques de propiedad pública (SFB 2021). Asimismo, cuenta con el Instituto Brasileño del Medio Ambiente y de los Recursos Naturales Renovables, creado en 1989, vinculado al Ministerio de Medio Ambiente, que ejerce el control y fiscalización sobre el uso, manejo y transporte de los recursos forestales por medio de su Dirección de Uso Sustentable. La gestión forestal en los bosques públicos en Brasil se realiza a través de una gestión directa sobre los bosques nacionales, estaduales (regional) y municipales (local) (IBAMA 2021).

En tal sentido, si bien en Perú, Brasil y Colombia la gestión de los RFFS es descentralizada los modelos y entidades concentran funciones de forma diferente respecto al caso peruano; como similitud, existe un ente rector que da directrices y un nivel administrativo menor es quien las opera. En el caso se Brasil la gestión de los recursos forestales abarca los tres niveles de Gobierno a diferencia del caso peruano y colombiano. Sin embargo, en los casos de Colombia y Brasil, no se observa la heterogeneidad en cuanto a la gestión de los RFFS que se observa en Perú, con parte del país en un modelo descentralizado y otra parte desconcentrado con dependencia administrativa de SERFOR.

\section{Evaluación del ejercicio de las entidades en- cargadas de la administración y control de los RFFS}

Las ATFFS en el ámbito de los departamentos de Cajamarca, Cusco cuentan con bajos presupuestos, pese a que la gestión de los RFFS en ambos departamentos se realiza a través de una ATFFS. Esto, se debería al poco interés del SERFOR en mantener una gestión desconcentrada, que no articula con sus funciones, ni con la organización establecida en la LFFS y sus Reglamentos. El mínimo presupuesto ejecutado en Pasco se debería a que el SERFOR no gestiona los RFFS de Pasco a través de una ATFFS; sino que junto a Junín conforman la ATFFS Selva Central.

En el caso de las ARFFS de Loreto y Ucayali el presupuesto ejecutado en el periodo 20122018 no superó el 1\% respecto al total ejecutado por dichos Gobiernos Regionales; si bien es cierto, en el periodo evaluado ambos Gobiernos Regionales incrementaron su ejecución, esta tendencia no se siguió en sus ARFFS que 
disminuyeron su ejecución. Comparando la ejecución de ambas ARFFS se puede afirmar que Ucayali destina mayores recursos en la gestión de los RFFS que su similar de Loreto; lo cual se evidencia en la producción de madera rolliza (m3) del año 2019 donde Ucayali (448 $656,42 \mathrm{~m} 3$ ) supera a su similar de Loreto (338 $750,76 \mathrm{~m} 3$ ), acaparando el $38 \%$ de la producción, mientras que Loreto representa solo el $26 \%$ de la producción (SERFOR 2020).

Los bajos porcentajes de presupuesto ejecutado en el PP Forestal en Cajamarca, Cusco y Pasco, da cuenta del poco interés por parte del SERFOR en destinar recursos hacia estos departamentos en el marco del PP forestal. A pesar, de que el SERFOR es responsable del PP forestal y las ATFFS de estos departamentos se encuentran bajo su dependencia.

La ejecución de la ARFFS Loreto en el PP forestal es irrelevante, considerando que cuenta con $52 \%$ de los bosques de producción permanente del país; y más de 45 millones de hectáreas de bosques naturales. Por otro lado, la ARFFS Ucayali demostró conocimiento de la herramienta de Presupuesto por resultados; toda vez que Ucayali, fue en el 2016 el segundo Gobierno Regional en ejecutar mayores recursos en el PP forestal, en el 2017 fue el Gobierno Regional que más recursos ejecutó en el PP forestal, y en el 2018 repitió el segundo lugar.

En relación a los PI se puede afirmar que tanto en las ATFFS, como en las ARFFS se determinó la incipiente implementación de esta herramienta. Sin embargo, de acuerdo con la información revisada se puede afirmar que las causas asociadas serían distintas; dado que, mientras en las ATFFS se puede afirmar que existe un nulo interés por parte de SERFOR en desarrollar esta herramienta en el mejoramiento de dichas ATFFS; en el caso de las ARFFS, se debería a la alta rotación de personal y al deficiente desarrollo de capacidades en los Gobiernos Regionales. En tal sentido, en este último, se debería a causas asociadas a problemas del propio proceso de descentralización a nivel nacional.

Con respecto al SCI, si bien el SERFOR y el Gobierno Regional de Ucayali están en un nivel de maduración intermedio, no se puede afirmar que no existan procesos en su gestión que cuenten con débiles controles y que son susceptibles de prácticas corruptas. Este riesgo se incrementa en el caso del Gobierno Regional de Loreto que se encuentra en un nivel inicial. Cabe indicar, que en ambos casos existen evidencias de prácticas corruptas en la gestión forestal que han sido reveladas por entidades como el OSINFOR (OSINFOR 2015) y la CGR (CGR 2015b).

\section{Análisis de deficiencias de informes de con- trol sobre la gestión de los RFFS}

\section{Deficiencias de las ATFFS seleccionadas}

Las deficiencias identificadas a partir de la gestión de las ATFFS son consecuentes con las funciones que vienen ejerciendo; en tal sentido, es alarmante que el mayor número de deficiencias esté relacionado al incumplimiento de procedimientos técnicos de aprovechamiento de recursos forestales; toda vez, que esto podría ocasionar la extracción irregular de RFFS, o su sobreexplotación. Por otro lado, también se incumplen procedimientos sancionadores; pese a que ello, coadyuvaría a lograr un elemento disuasivo frente a actos irregulares.

Adicionalmente, se observó que en las ATFFS existen debilidades concernientes a la documentación y archivo de derechos de aprovechamiento; esta situación, configura un escenario perfecto para no advertir la falta de presentación de requisitos; así como que los hechos actuados irregularmente no dejen evidencias documentales. También, se observó el deficiente manejo de sus bases de datos en lo correspondiente a los volúmenes de madera que aprovechan los titulares de permisos, concesiones o autorizaciones; esto limita dos aspectos: la correcta emisión de GTF; y el control del transporte que realizan estas ATFFS.

Las deficiencias de las ATFFS en lo concerniente a sus controles y revisiones, están relacionadas al inadecuado control de las GTF que emiten, la ausencia de inspecciones oculares previas o posteriores a títulos los habilitantes otorgados para el aprovechamiento forestal, y las limitadas actividades de supervisión del 
transporte de productos forestales. Además, se evidenció una limitada supervisión y evaluación al accionar de las ATFFS por parte del SERFOR, situación que de remediarse podría coadyuvar a que las ATFFS cumplan eficientemente sus labores de control y vigilancia al aprovechamiento y transporte de los RFFS y productos.

\section{Deficiencias de las ARFFS seleccionadas}

Las deficiencias respecto a la gestión de los RFFS a cargo de los Gobiernos Regionales de Loreto y Ucayali evidenciaron principalmente deficiencias de ejecución y funcionamiento, debido al incumplimiento de procedimientos en el otorgamiento de permisos de exportación de las especies maderables de Caoba y Cedro, en la evaluación y otorgamiento de permisos de aprovechamiento forestal, en el tratamiento de productos forestales maderables incautados, en la ejecución de procedimientos sancionadores, así como la emisión de GTF cuando no correspondía.

Asimismo, en cuanto a las deficiencias de controles y revisiones se observó la ausencia de supervisión a las actividades de extracción forestal de concesiones forestales, la deficiente verificación en campo de la información consignada en los planes de manejo forestal de los permisos y concesiones forestales previo a su aprobación, la irregular emisión de GTF; así como, la falta de mecanismos para el control forestal de productos forestales y RFFS, que permita realizar incautaciones.

Cabe precisar, que desde el 2015 hasta el 2020 el SERFOR ejecutó S/92 000000 con recursos que provenían de donaciones y operaciones oficiales de crédito, provenientes en su mayoría de la cooperación internacional (Agencias Internacionales de Desarrollo y Organismos Internacionales) (MEF 2021). Del total, aproximadamente S/ 36000000 se destinaron en proyectos y actividades que en parte involucraban el control forestal (trazabilidad, acceso a información; entre otros) en departamentos amazónicos (MEF 2021).

Por otro lado, con recursos de USAID Perú, a través del Proyecto Perú Bosques se culminó la implementación del Sistema Nacional de Información Forestal y de Fauna Silvestre- SNIFFS (CGR 2017); cuyo Módulo de Control en la actualidad no se encuentra operativo. Al respecto agregar que el funcionamiento del Módulo de Control del SNIFFS fue observado por la CGR en el 2017, advirtiéndose limitaciones en su operatividad, fallas del software, registro de información incompleta; así como, que SERFOR no tenía previsto en su planeamiento institucional, ni en su presupuesto institucional la permanencia del SNIFFS (CGR 2017).

Es importante resaltar que el proyecto para la implementación del SNIFFS se comenzó a ejecutar bajo el nombre de Sistema Nacional de Información y Control Forestal y de Fauna Silvestre- SNIC desde el año 2012, durante la gestión de la extinta DGFFS; y desde esa fecha la autoridad nacional forestal viene recibiendo recursos para el funcionamiento de dicha herramienta que contribuiría al control forestal y por consiguiente un arma importantísima corrupción en el sector forestal.

\section{Análisis FODA e Identificación de Riesgos}

Producto del Análisis FODA se pudo identificar acciones que deberían implementar las ATFFS continúen bajo su dependencia; SERFOR: i) implementar el control de las GTF que emiten, ii) realizar inspecciones oculares a títulos habilitantes otorgados y establecer mecanismos de supervisión del transporte de productos forestales. Así como, fortalecer la gestión de la información (inventarios, diagnósticos, archivos, registros, bases de datos). Adicionalmente, el SERFOR debe realizar una supervisión del cumplimiento de los procedimientos sancionadores o técnicos, y culminar la implementación del SCI.

Por otro lado, el FODA de las ARFFS permitió identificar acciones como: i) Fortalecer las capacidades de su personal, en PP y PI; ii) Priorizar PI enfocados al mejoramiento de la infraestructura para el control y vigilancia del transporte de productos forestales; iii) Establecer mecanismos de control sobre el cumplimiento de los procedimientos administrativos, sancionadores y técnicos; iv) Establecer 
la obligatoriedad de realizar inspecciones oculares previas o posteriores a la aprobación de planes de manejo forestal; así como, de realizar periódicamente el control del transporte de los productos forestales; y, v) Fortalecer su coordinación interinstitucional, materializando el intercambio de información con la Policía $\mathrm{Na}$ cional, las FEMAs, el OSINFOR; y, el MINAM.

Cabe indicar que las debilidades y amenazas de las ARFFS y ATFFS fueron la base para la identificación de riesgos. Los riesgos identificados, son equivalentes para las ATFFS y ARFFS; debido a que los factores que afectan a los procesos de gestión de los RFFS son equivalentes en todo el territorio nacional. No obstante, la valoración de los riegos para cada caso particular está asociadas a las presiones de cada grupo, en función a sus características específicas.

\section{Diagrama de Verificación de Riesgos}

A partir del diagrama de identificación de riesgos se evidenció que los riesgos en que se encuentran los RFFS en cuanto a su probabilidad de ocurrencia e impacto, son mayores para las ARFFS al albergar en mayor cantidad y riqueza los RFFS a nivel nacional. Esto se debe a las deficiencias propias del proceso de descentralización a nivel nacional; es decir, sin trasferencia de recursos económicos, logístico, y humanos.

\section{Conclusiones}

La descentralización de funciones forestales a los Gobiernos Regionales, en el periodo 2012-2018, no representó un avance en la gestión de los RFFS; considerando el presupuesto, programas presupuestales e inversión pública; así como, las deficiencias de ejecución $\mathrm{y}$ funcionamiento; $\mathrm{y}$, controles y revisiones en sus procesos.

Las ATFFS presentan deficiencias que radican en el poco interés del SERFOR en fortalecer su gestión, cuentan con un escaso presupuesto, incipiente participación en el presupuesto por resultados y PI que SERFOR ejecuta. A nivel normativo, el carácter temporal de las ATFFS contemplado en la LFFS N² 29763.
Las ARFFS presentan deficiencias relacionadas al escaso presupuesto que destinan los Gobiernos Regionales en su funcionamiento, limitada ejecución de PI; así como, la falta de capacidad técnica.

SERFOR es el actor más interesado en culminar la transferencia de funciones forestales; mientras, que las ATFFS y los Gobiernos Regionales, que darían y recibirían las funciones, estarían en contra.

Los riesgos sobre los RFFS en el ámbito de las ARFFS y ATFFS son equivalentes; sin embargo, la probabilidad e impacto es mayor para las ARFFS al albergar en mayor cantidad y riqueza los RFFS a nivel nacional.

\section{Agradecimientos}

Al Ministerio de Economía y Finanzas y a la Contraloría General de la República por poner a la disposición de la ciudadanía sus cifras e informes de control; respectivamente.

\section{Referencias}

Arredondo, CD. 2011. La descentralización en Chile: una mirada desde la economía política y el neoinstitucionalismo. Tesis Mg. Santiago de Chile, Chile, Universidad de Chile. 96 p.

Ley de Bases de la Descentralización n. 27783. Diario Oficial El Peruano. Perú.17 jul. 2002.

CGR (Contraloría General de la República, Perú). 2014. Estudio del proceso de descentralización en el Perú. Lima, Perú. 415 p.

CGR (Contraloría General de la República, Perú). 2015a. Medición de la Implementación del Sistema de Control Interno en el EstadoAño 2014. Lima. 62 p.

CGR (Contraloría General de la República, Perú). 2015b. Informe de Auditoría de Cumplimiento N568-2015-CG/MA-AC "Emisión de GTF por el Programa Regional de Manejo de Recursos Forestales y de Fauna Silvestre del Gobierno Regional de Loreto" (en línea).Lima. Perú. Consultado 2 abr. 2021. Disponible en https://apps1.contraloria.gob.pe/portal/BuscadorInformes/BuscadorInformes2.htm. 
CGR (Contraloría General de la República, Perú). 2017. Informe de Acción Simultánea N657-2017-CG/L215-AS "Implementación del Módulo de Control del Sistema Nacional de Información Forestal y de Fauna Silvestre" (en línea). Consultado 2 abr. 201. Disponible en http://lfweb.contraloria.gob.pe/BuscadorInformes/DocView.aspx?id=4264138\&cr=1.

CGR (Contraloría General de la República, Perú). 2018. Consulta efectuada sobre la madurez del Sistema de Control Interno de SERFOR y de los Gobiernos Regionales de Loreto y Ucayali. Lima, Perú. 3p. Oficio n. ${ }^{\circ}$ 526-2018CG/SGE.

CGR (Contraloría General de la República, Perú). 2019. Búsqueda de informes de control (en línea). Lima, Perú. Consultado 30 dic 2019. Disponible en https://apps1.contraloria. gob.pe/portal/BuscadorInformes/BuscadorInformes.htm.

DP (Defensoría del Pueblo, Perú). 2018. Adjuntía para la prevención de conflictos sociales y la gobernabilidad (en línea). Lima, Perú. 110 p. Reporte de conflictos sociales n. ${ }^{\circ} 178$. Consultado 20 ene. 2019. Disponible en https://www.defensoria.gob.pe/wp-content/uploads/2019/01/ Conflictos-Sociales-N\%C2\%B0-178-Diciembre-2018.pdf.

DAFP (Departamento Administrativo de la Función Pública). 2018. Estructura del Estado Colombiano: 27 CORPORACIONES AUTÓNOMAS REGIONALES Y DE DESARROLLO SOSTENIBLE. (en línea). Bogotá, Colombia. Consultado 30 mar. 2021. Disponible en http://www.cornare.gov.co/Estrategico/ estructura-corporaciones.pdf.

INEI (Instituto Nacional de Estadística e Informática, Perú). 2016. Informe técnico: evolución de la pobreza monetaria 2009-2015. Lima, Perú.159 p.

IBAMA (Instituto Brasileño del Medio Ambiente y de los Recursos Naturales Renovables). 2021. Composición (en línea). Brasilia, Brasil. Consultado 15 mar. 2021. Disponible en https://www.gov.br/ibama/pt-br/composicao/ quem-e-quem.
MEF (Ministerio de Economía y Finanzas, Perú). 2021. Consulta amigable: ejecución del gasto de SERFOR por fuente de financiamiento (en línea). Lima, Perú. Consultado 2 abr. 2021. Disponible en https://apps5.mineco. gob.pe/transparencia/Navegador/default.aspx$? \mathrm{y}=2020 \& a p=$ ActProy.

MEF (Ministerio de Economía y Finanzas, Perú). 2019a. Consulta amigable: ejecución del gasto por pliego y departamento (en línea). Lima, Perú. Consultado 18 set. 2019. Disponible en http://apps5.mineco.gob.pe/transparencia/ Navegador/default.aspx.

MEF (Ministerio de Economía y Finanzas, Perú). 2019b. Consulta amigable: ejecución del gasto por programa presupuestal y entidad (en línea). Lima, Perú. Consultado 18 set. 2019. Disponible en http://apps5.mineco.gob.pe/transparencia/Navegador/default.aspx.

MINAGRI (Ministerio de Agricultura y Riego, Perú). 2017. Plan anual de transferencia de competencias sectoriales a los gobiernos regionales y locales del año 2017. Lima, Perú. 14 p.

MINAGRI (Ministerio de Agricultura y Riego, Perú). 2019. Plan anual de transferencia de competencias sectoriales a los gobiernos regionales y locales del año 2019. Lima, Perú. 20 p.

MINAM (Ministerio del Ambiente, Perú). 2015a. Cuantificación y análisis de la deforestación en la Amazonia peruana en el periodo 2010-2011-2013-2014 (en línea). Lima, Perú. Consultado 30 ene. 2020. Disponible en http://infobosques.com/portal/wp-content/ uploads/2017/03/Memoria Descriptiva Cambios Cobertura Bosque 2014.pdf.

MINAM (Ministerio del Ambiente, Perú). 2015b. Mapa nacional de cobertura vegetal: memoria descriptiva (en línea). Lima, Perú. 100 p. Consultado 10 ene. 2020. Disponible en http://www.minam.gob.pe/patrimonio-natural/wp-content/uploads/sites/6/2013/10/MAPA-NACIONAL-DE-COBERTURA-VEGETAL-FINAL.compressed.pdf.

MINAM (Ministerio del Ambiente, Perú). 2017. Estado situacional de la Zonificación 
Ecológica y Económica (ZEE) a nivel regional: enero 2017 (en línea, infografía). Lima, Perú. 1 infografía, color. Consultado 21 ene. 2020. Disponible en http://www.minam.gob.pe/ordenamientoterritorial/wp-content/uploads/ sites/129/2017/02/Avances-en-regiones-ZEEMapa.pdf.

MINAMBIENTE (Ministerio del Ambiente y Desarrollo Sostenible, Colombia).2021. Organigrama. (en línea). Bogotá, Colombia. Consultado 15 mar. 2021. Disponible en https://www. minambiente.gov.co/index.php/ministerio/ organigrama.

OSINFOR (Organismo de Supervisión de los Recursos Forestales y de Fauna Silvestre, Perú). 2016. Sistema de información gerencial del OSINFOR: volumen movilizado proveniente de extracción ilegal 2011-2016 (en línea). Lima, Perú. Consultado 30 dic. 2017. Disponible en https://observatorio.osinfor.gob.pe/Estadisticas/Home/Reportes/12.

OSINFOR (Organismo de Supervisión de los Recursos Forestales y de Fauna Silvestre, Perú).2015. Resultados de las supervisiones y fiscalizaciones efectuadas por el OSINFOR en el marco del Operativo Internacional "Operación Amazonas 2014". Lima. 63 p.

PNUD (Programa de las Naciones Unidas para el Desarrollo, Estados Unidos de América). 2013. Informe sobre desarrollo humano Perú 2013. Lima, Perú. 205 p.

SERFOR (Servicio Nacional Forestal, Perú).2020. Anuario Forestal y de Fauna Silvestre 2019 (en línea).Lima, Perú. Consultado el 2 abr. 2021. Disponible en http://repositorio. serfor.gob.pe/handle/SERFOR/408.

SFB (Servicio Forestal Brasileño). 2021. Institucionalidad. Brasilia, Brasil. Consultado 15 mar. 2021. Disponible en https://www.florestal. gov.br/institucional.

TCU (Tribunal de Cuentas de la Unión, Brasil). 2002. Técnica de auditoría "Análisis de Stakeholders". Boletim do Tribunal de Contas da União 35(13):1-20.

Vidal, JN. 2004. Descentralización: un proceso institucional para mejorar el desempeño gu- bernamental local en México. Tesis Mg. Nuevo León, México, UANL. 58p. 